\title{
Consistent Quantum Measurements
}

\author{
Robert B. Griffiths * \\ Department of Physics, Carnegie-Mellon University, \\ Pittsburgh, PA 15213, USA
}

Version of 26 June 2015

\begin{abstract}
In response to recent criticisms by Okon and Sudarsky, various aspects of the consistent histories $(\mathrm{CH})$ resolution of the quantum measurement problem(s) are discussed using a simple Stern-Gerlach device, and compared with the alternative approaches to the measurement problem provided by spontaneous localization (GRW), Bohmian mechanics, many worlds, and standard (textbook) quantum mechanics. Among these $\mathrm{CH}$ is unique in solving the second measurement problem: inferring from the measurement outcome a property of the measured system at a time before the measurement took place, as is done routinely by experimental physicists. The main respect in which $\mathrm{CH}$ differs from other quantum interpretations is in allowing multiple stochastic descriptions of a given measurement situation, from which one (or more) can be selected on the basis of its utility. This requires abandoning a principle (termed unicity), central to classical physics, that at any instant of time there is only a single correct description of the world.
\end{abstract}

Keywords: Quantum measurement; consistent histories; unicity; quantum property

\section{Introduction}

The immediate motivation for this paper comes from criticisms by Okon and Sudarsky [1], recently published in this journal, of the consistent histories $(\mathrm{CH})$ interpretation of quantum mechanics. These authors claim that $\mathrm{CH}$ does not provide a satisfactory resolution of the quantum measurement problem. Such criticism deserves to be taken seriously, for the $\mathrm{CH}$ approach claims to resolve all the standard problems of quantum interpretation which form the bread and butter of quantum foundations research: it is local [2], so there are no conflicts with special relativity; it is noncontextual [3], in contrast to hidden variables interpretations; it resolves the EPR, BKS, Hardy, three boxes, etc., etc. paradoxes, see Chs. 19-25 of [4]. And while it may be defective, its (purported) solutions to the full gamut of quantum conceptual difficulties have been published in detail and are available right now for critical inspection, not just as promissory notes for some future time. Thus the Okon and Sudarsky criticisms, while based (we believe) on an imperfect understanding of the $\mathrm{CH}$ approach, are dealing with important issues that need to be discussed.

Of particular significance is the fact that the $\mathrm{CH}$ approach does not include any reference to measurements among its basic principles for interpreting quantum mechanics. Measurements are simply treated as a particular type of physical process to which the same quantum principles apply as to any other physical process. When understood in this way quantum mechanics no longer has a measurement problem as that term is generally used in quantum foundations: a conflict between unitary time development of a combined system plus measuring device and a macroscopic outcome or "pointer position." Not only so, in addition $\mathrm{CH}$ shows how the outcome of a measurement can be shown to reveal the presence of a microscopic quantum property possessed by the measured system just before the measurement took place, in accordance with the belief, common among experimental physicists, that the apparatus they have built performs the function for which it was constructed. This second measurement problem has received far too little attention in the quantum foundations literature, and resolving it is no less important than the first problem if the entire measuring process is to be understood in fully quantum-mechanical terms.

*Electronic mail: rgrif@cmu.edu 
Rather than an abstract discussion, the present paper examines a particular measurement scenario, using it as an example of the application of $\mathrm{CH}$ principles, and also a basis for comparison with some other interpretations of quantum mechanics mentioned in [1]. These include the spontaneous localization approach developed by Ghirardi et al. and Pearle, see [5-9], often abbreviated as GRW (the initials of the authors of [5]), and the pilot wave approach of de Broglie and Bohm, which we shall refer to as Bohmian mechanics [10-13]. Textbook or standard quantum mechanics and the many worlds interpretation of Everett and his successors, [14-16] also enter the discussion from time to time. Since details of the CH approach are readily available in the literature, e.g., [4,17-21], only those aspects needed to make the discussion reasonably self-contained are included in this paper.

Our aim is to present and discuss as clearly as possible the central features of the $\mathrm{CH}$ approach that have given rise to the criticisms in [1], and which are undoubtedly shared by other critics, e.g., [22-25]. Of particular importance is the fact that $\mathrm{CH}$ abandons a principle, here called unicity, which is deeply embedded in both conventional and scientific thought, and is taken for granted in classical physics. It is the idea that at any instant of time there is precisely one exact description of the state of the world which is true. If the $\mathrm{CH}$ understanding is correct, quantum mechanics has made unicity obsolete in somewhat the same way as modern astronomy has replaced an unmovable earth at the center of the universe with our current understanding of the solar system, and ignoring this feature of the quantum world is what has given rise to so many conceptual difficulties.

The contents of the remainder of the paper are as follows. The measurement problem(s) of quantum foundations are discussed in general terms in Sec. 2, followed in Sec. 3 by a specific measurement model, a modernized version of the famous experiment of Stern and Gerlach [26,27]. Its description in CH terms begins in Sec. 4 with a discussion of the first measurement problem, whose solution is compared with some other approaches in Sec. 4.2. The CH solution to the second measurement problem is the subject of Sec. 5, and it is compared with standard quantum mechanics, spontaneous localization, many worlds, and Bohmian mechanics in Sec. 6. Our response to the specific criticisms of Okon and Sudarsky occupies Sec. 7. The concluding Sec. 8 is a brief summary of the whole paper.

\section{The Quantum Measurement Problem}

Physics is an experimental science, and measurements and observations play a central role in testing the empirical contents of its theories. This was also the case before the quantum revolution of the twentieth century, and yet classical physics had no measurement problem. Why, then, is the measurement problem considered the central issue in quantum foundations, the one that must be resolved if progress is to be made in this field? The essence of the measurement problem is easy to state. If quantum mechanics applies not only to the microscopic world of nuclei and atoms, but also to macroscopic objects and things that are even larger - from the quarks to the quasars - then the measurement process in which an earlier microscopic property is revealed in a macroscopic outcome should itself be describable, at least in principle, in fully quantum mechanical terms. Applied equally to the system being measured and to the macroscopic apparatus, and without the evasion and equivocation ridiculed by Bell [28]. It is indeed a scandal that the quantum physics community has not been able to agree on a solution to this problem. Would not the stories told by modern cosmologists be dismissed as pure fantasy if astronomers did not understand the operation of their telescopes?

It is useful to separate the general quantum measurement problem into two parts. The better known first measurement problem arises when the initial state of the measured system - hereafter for convenience thought of as a particle - is such that the unitary time development resulting from coupling it to a measurement apparatus results in a superposition of two or more states in which the apparatus pointer (in the archaic but picturesque language of quantum foundations) points in different directions. How is this "Schrödinger cat" to be interpreted, given that in the laboratory the pointer always points in a definite direction? The second measurement problem is to explain how the actual (single) pointer direction is related to the property of the particle the apparatus was designed to measure, at a time before the measurement took place? Unfortunately, many textbooks speak of a "measurement" not as revealing a pre-existing property, but as a correlation between the pointer and the particle after the measurement has taken place. The latter should be called a preparation rather than a measurement; for a discussion of this from the $\mathrm{CH}$ perspective see Sec. 3.5 of [20] and Sec. 7.3 of [21].

It is perhaps worth mentioning that in textbooks probabilities are introduced in connection with mea- 
surements, and not as a separate topic. As a consequence the perplexities associated with an unresolved measurement problem are transferred to an inconsistent discussion of probabilities. Thus cleaning up the quantum measurement problem is intimately connected with introducing probabilities in quantum mechanics in a consistent way, not associated with measurements, something which is not present in any textbook of which we are aware.

\section{Stern Gerlach Spin Measurement}

\subsection{Description}

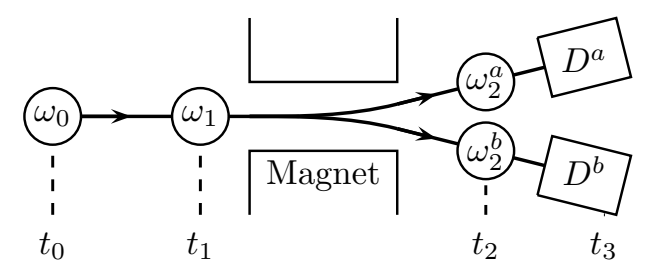

Figure 1: Stern Gerlach apparatus for measuring spin half

Figure 1 is a schematic diagram of a Stern Gerlach device to measure the spin of a spin-half particle. The particle arrives from the left and its initial state at time $t_{0}$ is $\left|\omega_{0}\right\rangle \otimes\left|\chi_{0}\right\rangle$, where $\left|\omega_{0}\right\rangle$ refers to its position, corresponding to a wavepacket $\omega_{0}(\mathbf{r})=\left\langle\mathbf{r} \mid \omega_{0}\right\rangle$, and $\left|\chi_{0}\right\rangle$ denotes the spin, with $\left|z^{+}\right\rangle$and $\left|z^{-}\right\rangle$the eigenstates of $S_{z}$. The unitary time development of the particle state at successive times $t_{0}<t_{1}<t_{2}$ as it passes through the magnetic field gradient is given by:

$$
\left|\omega_{0}\right\rangle \otimes\left|z^{+}\right\rangle \rightarrow\left|\omega_{1}\right\rangle \otimes\left|z^{+}\right\rangle \rightarrow\left|\omega_{2}^{a}\right\rangle \otimes\left|z^{+}\right\rangle ; \quad\left|\omega_{0}\right\rangle \otimes\left|z^{-}\right\rangle \rightarrow\left|\omega_{1}\right\rangle \otimes\left|z^{-}\right\rangle \rightarrow\left|\omega_{2}^{b}\right\rangle \otimes\left|z^{-}\right\rangle
$$

where $\left|\omega_{j}\right\rangle$ gives the (approximate) location of the particle at time $t_{j}$. The trajectories of a particle with $S_{z}=+1 / 2$ and one with $S_{z}=-1 / 2$ are initially identical, but at time $t_{2}$ there is a small but macroscopic separation between the wave packet $\omega_{2}^{a}(\mathbf{r})$, the particle moving upwards towards detector $D^{a}$, and $\omega_{2}^{b}(\mathbf{r})$, the particle moving downwards towards detector $D^{b}$. By time $t_{3}$ the detector $D^{a}$ will have triggered if the particle had $S_{z}=+1 / 2$, and $D^{b}$ it the particle had $S_{z}=-1 / 2$. We assume these detectors are capable of detecting individual atoms, as is possible nowadays by first ionizing the atom and then using an electron multiplier to convert the emerging electron into a macroscopic current pulse.

Now let us treat the detectors as quantum objects by assigning kets $\left|D^{a}\right\rangle,\left|D^{b}\right\rangle$ for their initial (ready) states, and $\left|D^{a *}\right\rangle$ and $\left|D^{b *}\right\rangle$ if the detector has been triggered by absorbing the particle. If we assume that at $t_{0}$ the spin state of the particle is

$$
\left|\chi_{0}\right\rangle=\alpha\left|z^{+}\right\rangle+\beta\left|z^{-}\right\rangle
$$

the unitary time development of the detectors along with the particle, regarded as a single closed quantum system, is given by

$$
\left|\Psi_{0}\right\rangle \rightarrow\left|\Psi_{1}\right\rangle \rightarrow\left|\Psi_{2}\right\rangle \rightarrow\left|\Psi_{3}\right\rangle
$$

where

$$
\begin{aligned}
& \left|\Psi_{j}\right\rangle=\left|\omega_{j}\right\rangle \otimes\left(\alpha\left|z^{+}\right\rangle+\beta\left|z^{-}\right\rangle\right) \otimes\left|D^{a}\right\rangle \otimes\left|D^{b}\right\rangle \text { for } j=0,1 ; \\
& \left|\Psi_{2}\right\rangle=\left(\alpha\left|\omega_{2}^{a}\right\rangle \otimes\left|z^{+}\right\rangle+\beta\left|\omega_{2}^{b}\right\rangle \otimes\left|z^{-}\right\rangle\right) \otimes\left|D^{a}\right\rangle \otimes\left|D^{b}\right\rangle ; \\
& \left|\Psi_{3}\right\rangle=\alpha\left|D^{a *}\right\rangle \otimes\left|D^{b}\right\rangle+\beta\left|D^{a}\right\rangle \otimes\left|D^{b *}\right\rangle,
\end{aligned}
$$

(Notice that there is no separate particle state at $t_{3}$, as the particle has been absorbed into the detector and its spin state is no longer relevant.)

\subsection{Discussion}

The (first) measurement problem of quantum foundations is apparent in (6) in a situation in which both $\alpha$ and $\beta$ are nonzero, and thus $\left|\Psi_{3}\right\rangle$ is a superposition of two states which are macroscopically quite distinct: 
in one case $D^{a}$ has triggered, and in the other case $D^{b}$. There seems to be some sort of conflict between unitary time evolution and the existence of a definite measurement outcome in which either $D^{a}$ has triggered (as indicated by some pointer position) and $D^{b}$ remains in the ready state, or $D^{b}$ has triggered and $D^{a}$ has not.

How is this to be understood? Let us consider some of the better-known approaches to this problem, starting with standard or textbook quantum mechanics. Here the usual strategy is to give various reasons why "for all practical purposes" (6) is to be understood as telling us that with probability $|\alpha|^{2}$ detector $D^{a}$ has been triggered, and $D^{b}$ with probability $|\beta|^{2}$. For a detailed critique of this approach, see [28]. Few physicists working in the area of quantum foundations consider this solution satisfactory, even though it employs the sort of reasoning which is known to agree with laboratory experiments. Nowadays decoherence due to an environment is sometimes invoked in order to justify the this approach, but by itself it does not solve the problem, as the environment can always be included in the detector states $\left|D^{a}\right\rangle,\left|D^{a *}\right\rangle\left|D^{b}\right\rangle$, and $\left|D^{b *}\right\rangle$; see, for example [29].

In the spontaneous localization interpretation the unitary dynamics leading from $t_{2}$ to $t_{3}$, i.e., from (5) to (6), should be replaced by a stochastic dynamics in which, because detectors consist of a large number of particles, with some probability $\left|D^{a *}\right\rangle \otimes\left|D^{b}\right\rangle$, or at least some ket that represents the same macroscopic physics, will be the correct quantum state at time $t_{3}$, and with some probability it will be $\left|D^{a}\right\rangle \otimes\left|D^{b *}\right\rangle$. In Bohmian mechanics a collection of "hidden variables", particle positions, are added to the Hilbert space description, and thus whereas $\left|\Psi_{3}\right\rangle$ is the correct wave function at time $t_{3}$, the particles which make up the detectors will either correspond to the situation $\left|D^{a *}\right\rangle \otimes\left|D^{b}\right\rangle$ or to $\left|D^{a}\right\rangle \otimes\left|D^{b *}\right\rangle$, but not both; the part that does not correspond to the actual particle positions is considered an "empty wave." In the many worlds interpretation $\left|\Psi_{3}\right\rangle$ is the correct quantum state, but the two parts of it refer to what are, in effect, two different and unconnected "universes."

Once the first measurement problem has been solved and good reasons given why at the end of the experiment the pointer is in a well-defined position, which is to say one of the detectors in Fig. 1 has detected the particle and the other has not, the second measurement problem remains: what can we conclude about the quantum state of the particle before it was measured? In particular, if the final state is $\left|D^{a *}\right\rangle$, can we conclude that the particle's location corresponded to $\left|\omega_{2}^{a}\right\rangle$ at time $t_{2}$, rather than $\left|\omega_{2}^{b}\right\rangle$ ? Or that at $t_{1}$ the spin state was $\left|z^{+}\right\rangle$and not $\left|z^{-}\right\rangle$? In the world of particle physics experiments inferences of this sort are made all the time, and no interpretation of quantum mechanics which fails to provide a basis for them can be considered a satisfactory explanation of why quantum theory agrees with experiment.

\section{Consistent Histories and the First Measurement Problem}

\subsection{Quantum properties}

Before discussing how the situation in Fig. 1 is analyzed in the consistent histories (CH) approach it is useful to briefly review some of its key ideas. The first is that a physical property is always represented by a linear subspace of the Hilbert space used to describe the quantum system; equivalently, by the projector (orthogonal projection operator) onto this subspace. As an example, for a spin half particle $\left[z^{+}\right]=\left|z^{+}\right\rangle\left\langle z^{+}\right|$ is the projector onto the one-dimensional subspace or ray consisting of all multiples of $\left|z^{+}\right\rangle$, and corresponds to the property $S_{z}=+1 / 2$ in units of $\hbar$. (Here and later we use $[\psi]$ as an abbreviation for the projector $|\psi\rangle\langle\psi|$.) For a spinless particle in one dimension the subspace spanned by all wave packets $\psi(x)$ which are zero outside the interval $x_{1} \leq x \leq x_{2}$ corresponds to the property that the particle is located between $x_{1}$ and $x_{2}$.

A projector is the quantum analog of an indicator function $P(\gamma)$ for a classical property: $P(\gamma)$ is 1 at all points $\gamma$ in the phase space $\Gamma$ where the property is true, and 0 elsewhere. Indicator functions provide a convenient algebra for discussing classical properties. Thus if $I$ is the identity, the function taking the value 1 for all $\gamma$, the negation of a property $P$, "NOT $P$ ", has an indicator $I-P$; while if $P$ and $Q$ represent two properties, $P Q$ is the indicator for the conjunction " $P$ AND $Q$ ", and $P+Q-P Q$ the indicator for the disjunction " $P$ OR $Q$ ". Replacing classical indicators with quantum projectors leads to identical expressions for the algebra of quantum properties as long as the projectors $P$ and $Q$ are compatible, meaning they commute with each other, in which case $P Q=Q P$ is again a projector and can be interpreted as the property " $P$ AND $Q$ ".

However, when $P$ and $Q$ do not commute, the products $P Q$ and $Q P$ are not projectors, so neither can 
represent the conjunction. There are at least three approaches to this difficulty. One is to ignore it, a route leading to many quantum paradoxes. Another is to use the system of quantum logic initiated by Birkhoff and von Neumann [30], in which the conjunction of two properties is represented by the intersection of the corresponding subspaces, and the rules of propositional logic must be modified; see Sec. 4.6 of [4] for a simple example. A third approach is that of $\mathrm{CH}$ : the conjunction of properties represented by two incompatible projectors is undefined, "meaningless," and likewise the disjunction.

This leads to the single framework rule (SFR), a central principle of $\mathrm{CH}$, and one that is often misunderstood. It says that any valid quantum description of a physical system, i.e., any way of assigning it properties which may be either true or false, should be based upon a projective decomposition of the identity (PDI), a collection $\left\{P_{j}\right\}$ of mutually-orthogonal nonzero projectors that sum to the identity:

$$
I=\sum_{j} P_{j} ; \quad P_{j}=P_{j}^{\dagger}=P_{j}^{2} ; \quad P_{j} P_{k}=\delta_{j k} P_{j} .
$$

The orthogonality of the projectors ensures that the properties they represent are mutually exclusive alternatives: if one is true the others must be false. That they sum to the identity means that one (and only one) must be true. Thus these properties form a sample space as that term is used in probability theory. They, along with the projectors formed by taking sums of two or more of the $P_{j}$ 's, constitute an event algebra, which in the histories approach is called a framework (a term which is also used for the sample space). Probabilities can then be associated with events in the usual way: given a collection $\left\{p_{j}\right\}$ of nonnegative numbers summing to 1 , the probability of $P_{j}$ is $p_{j}$, and of $P_{2}+P_{3}$ is $p_{2}+p_{3}$, etc. In situations in which a single property $P$ is under discussion and a framework has not (yet) been defined, the implicit framework is $\{P, I-P\}$.

In the case of a spin-half particle the PDI associated with $S_{x}$ is $I=\left[x^{+}\right]+\left[x^{-}\right]$, and that associated with $S_{z}$ is $I=\left[z^{+}\right]+\left[z^{-}\right]$. However $\left[x^{+}\right]$and $\left[z^{+}\right]$do not commute, so a spin-half particle can never be said to be in a state with both $S_{z}=+1 / 2$ and $S_{x}=+1 / 2$. This is consistent with the observation that every ray in this two-dimensional Hilbert space is associated with $S_{w}=+1 / 2$ for some direction $w$ in space; there are no possibilities left over to express " $S_{x}=+1 / 2$ AND $S_{z}=+1 / 2$ "; there is no room in the Hilbert space for such a possibility. (For the quantum logic approach to this situation see Sec. 4.6 of [4].) Similarly " $S_{x}=+1 / 2$ OR $S_{z}=+1 / 2$ " makes no sense, because the disjunction must refer to projectors that commute with each other; otherwise they are not associated with a single PDI, and no logical comparison is possible.

It is important to note that the SFR is not a prohibition against constructing many incompatible descriptions using various incompatible frameworks. The theoretical physicist has perfect liberty to make up as many as desired. What the SFR prohibits is combining incompatible frameworks to make up a single description or carry out a single logical argument in the manner which, for example, leads to a Kochen-Specker paradox (see the discussion in Ch. 22 of [4]). Given two PDIs $\left\{P_{j}\right\}$ and $\left\{Q_{k}\right\}$, if every $P_{j}$ commutes with every $Q_{k}$ these sample spaces are compatible and possess a common refinement consisting of all nonzero products $P_{j} Q_{k}$. In this situation one can circumvent the single framework rule by using this common refinement to build a quantum description. But if the frameworks are not compatible the corresponding descriptions cannot be combined. Additional details of how the SFR governs quantum descriptions and reasoning are found in Ch. 16 of [4].

\subsection{Measurement outcomes}

Let us apply the foregoing discussion to the measurement situation shown in Fig. 1. The CH strategy is to first identify the quantum properties, which is to say the subspaces of the Hilbert space, that should enter the description. Here we are interested in a measurement with two possible outcomes: either detector $a$ has triggered or $b$ has triggered. While the individual states $\left|D^{a *}\right\rangle \otimes\left|D^{b}\right\rangle$ and $\left|D^{a}\right\rangle \otimes\left|D^{b *}\right\rangle$ can be interpreted as possible outcomes, their macroscopic superposition $\left|\Psi_{3}\right\rangle$ in (6) cannot, and indeed the projector $\left[\Psi_{3}\right]$ does not commute with any of the projectors $\left[D^{a *}\right],\left[D^{a}\right],\left[D^{b *}\right]$ or $\left[D^{b}\right]$. (Our notation follows the usual physicist's convention that a projector $P \otimes I$ on a tensor product can be denoted by $P$.) Consequently, if we demand that $\left[\Psi_{3}\right]$ be a physical property at time $t_{3}$, this choice of framework (i.e., $\left[\Psi_{3}\right]$ and $I-\left[\Psi_{3}\right]$ ) will prevent us from discussing the situation in Fig. 1 as a measurement, a physical process with some specific macroscopic outcome. Instead we must use a framework at time $t_{3}$ that contains the projectors $\left[D^{a}\right],\left[D^{a *}\right]$, $\left[D^{b}\right],\left[D^{b *}\right]$ and their products. Having made this choice $\mathrm{CH}$ employs $\left|\Psi_{3}\right\rangle$ not as a physical property but as a pre-probability, a mathematical device which can be used to calculate the probability of various properties 
via the usual Born formula:

$$
\operatorname{Pr}\left(D^{a *}\right)=\left\langle\Psi_{3}\left|D^{a^{*}} \otimes I\right| \Psi_{3}\right\rangle=|\alpha|^{2}, \quad \operatorname{Pr}\left(D^{b *}\right)=|\beta|^{2}, \quad \operatorname{Pr}\left(D^{a *}, D^{b *}\right)=0 .
$$

(Here $\operatorname{Pr}(A, B)$ is the probability of the conjunction $A$ AND $B$.) In words, the probability is $|\alpha|^{2}$ that the $a$ detector has triggered, $|\beta|^{2}$ that the $b$ detector has triggered, and 0 that both have triggered. This way of understanding $\left|\Psi_{3}\right\rangle$ is not a new innovation, as it goes back to the work of Born in $1926^{1}$.

Thus the first measurement problem is resolved in the $\mathrm{CH}$ approach by employing a sample space that includes the final measurement outcomes (pointer positions) in its description, together with the unitary time development generated by the standard Schrödinger equation to assign probabilities using Born's formula. This is in contrast to the spontaneous localization approach which, as noted in Sec. 3.2, employs a stochastic modification of the Schrödinger equation. And it is quite unlike Bohmian mechanics in that no hidden variables are added to the Hilbert space description. Instead, $\mathrm{CH}$ uses the procedure students are actually taught in introductory quantum mechanics courses: compute the unitary evolution of a wave function and then use the result to calculate a probability (or an average) using Born's rule. The only difference is that the textbook may add a qualifying phrase to the effect that what has been calculated is the probability of something if it is measured. This qualification is not totally out of place, but it is also not particularly helpful, and would be unnecessary if the textbook included an appropriate discussion of quantum properties together with an explanation of how to make a consistent use of probabilities in the quantum context.

As noted above, in the $\mathrm{CH}$ discussion of measurement outcomes $\left|\Psi_{3}\right\rangle$ is not regarded as a quantum property, but instead as a pre-probability, a mathematical device employed to calculate probabilities, and hence no more "real" than a probability distribution of the sort one encounters in classical statistical mechanics. Indeed, it is perfectly possible to calculate the probabilities in (8) in a manner that never makes any reference to $\left|\Psi_{3}\right\rangle$; see the discussion in Sec. 9.4 of [4]. The CH approach stands in marked contrast with that of the many worlds interpretation, in which a unitarily evolving wave function, $\left|\Psi_{j}\right\rangle$ in our example, is regarded as describing what is really going on in the quantum world. But if $\left[\Psi_{3}\right]$ is a quantum property at time $t_{3}$ then from the $\mathrm{CH}$ perspective this precludes, invoking the SFR, a simultaneous assignment of pointer positions, i.e., specific detector states such as $\left[D^{a}\right]$ or $\left[D^{a *}\right]$, at time $t_{3}$. Thus $\mathrm{CH}$ and the many worlds approach are mutually incompatible.

\section{Consistent Histories and the Second Measurement Problem}

\subsection{Families of histories}

In order to understand how $\mathrm{CH}$ resolves the second measurement problem it is necessary to discuss its approach to the stochastic dynamics of a quantum system at a succession of times. It employs a sample space of histories, where a history is a sequence of (quantum) properties at a succession of times. For a sequence of times $t_{1}<t_{2}<\cdots t_{n}$ a typical history will have the form

$$
F_{1} \odot F_{2} \odot \cdots F_{n}
$$

where each $F_{j}$ is a projector corresponding to the quantum property at the time $t_{j}$. There is no assumption that the $F_{j}$ at successive times are related by the unitary time development produced by Schrödinger's equation. The symbol $\odot$ is a variant of the usual tensor product symbol $\otimes$, and indeed a collection or family of histories, also referred to as a framework or realm, can be thought of as represented as a decomposition of the identity of a history Hilbert space consisting of a tensor product of copies of the Hilbert space used for static properties. If such a family refers to events in a closed quantum system and satisfies certain consistency conditions it is possible to assign probabilities to the different histories using Schrödinger dynamics and an extension of the usual Born rule; the reader is referred to Sec. 3.4 of [19] for additional details.

\footnotetext{
${ }^{1}$ According to Jammer [31], p. 38, Born's first paper [32] proposing the probabilistic interpretation of Schrödinger's wave arrived at the publishers of Zeitschrift für Physik a mere four days after Schrödinger's concluding contribution (the part containing his time-dependent equation) had been sent to the editor of the Annalen der Physik. In a longer paper [33] published the same year Born stated (p. 804; translation from Jammer, op cit., p. 40): "The motion of particles conforms to the laws of probability, but the probability itself is propagated in accordance with the law of causality." Thus although Born did not use a technical term such as "pre-probability" for the wavefunction developing unitarily in time, the idea of employing it as a means of calculating probabilities was clearly present in his thinking. One wonders how the history of quantum foundations might have been different if the physics community, including Schrödinger, had taken Born's idea much more seriously, and proceeded to build on it.
} 
Let us consider the measurement process in Fig. 1 using a variety of different history frameworks, beginning with

$$
\mathcal{F}_{u}:\left[\Psi_{0}\right] \odot\left\{\left[\Psi_{1}\right], I-\left[\Psi_{1}\right]\right\} \odot\left\{\left[\Psi_{2}\right], I-\left[\Psi_{2}\right]\right\} \odot\left\{\left[\Psi_{3}\right], I-\left[\Psi_{3}\right]\right\}
$$

interpreted as follows: At $t=0$ the initial state is $\left[\Psi_{0}\right]=\left|\Psi_{0}\right\rangle\left\langle\Psi_{0}\right|$. At each later time $t_{j}$ there is a PDI which in this case is relatively simple: a choice between $\left[\Psi_{j}\right]$ and $I-\left[\Psi_{j}\right]$, i.e., either one of these or the other occurs or is true at the time in question. Thus the $\mathcal{F}_{u}$ family consists of $2^{3}=8$ different histories. But when probabilities are assigned to these histories using the extended Born rule, those containing an $I-\left[\Psi_{j}\right]$ are assigned 0 probability, and hence there would be no harm in omitting them. Thus in effect $\mathcal{F}_{u}$ contains only a single history representing unitary time evolution.

For reasons already discussed in Sec. $4.2, \mathcal{F}_{u}$ cannot represent a measuring process, as the use of $\left[\Psi_{3}\right]$ at $t_{3}$ precludes any discussion of measurement outcomes. A more satisfactory family from this perspective is

$$
\mathcal{F}_{1}:\left[\Psi_{0}\right] \odot\left[\Psi_{1}\right] \odot\left[\Psi_{2}\right] \odot\left\{\left[D^{a *}\right] \otimes\left[D^{b}\right],\left[D^{a}\right] \otimes\left[D^{b *}\right]\right\},
$$

where at times $t_{1}, t_{2}$ and $t_{3}$ projectors have been omitted if they only occur in histories having zero probability. An application of the extended Born rule assigns probabilities of $|\alpha|^{2}$ and $|\beta|^{2}$ to the histories terminating with the $a$ and $b$ detectors having detected the particle, in agreement with (8).

In order to describe properties of the particle before it was measured we introduce another family

$$
\mathcal{F}_{2}:\left[\Psi_{0}\right] \odot\left[\Psi_{1}\right] \odot\left\{\left[\omega_{2}^{a}\right],\left[\omega_{2}^{b}\right]\right\} \odot\left\{\left[D^{a *}\right] \otimes\left[D^{b}\right],\left[D^{a}\right] \otimes\left[D^{b *}\right]\right\} .
$$

Note that at $t_{2}\left[\omega_{2}^{a}\right]$ represents $\left[\omega_{2}^{a}\right] \otimes I \otimes I$ on the Hilbert space that includes the spin and the apparatus, and the same convention applies to $\left[\omega_{2}^{b}\right]$. Once again properties, such as $I-\left[\omega_{2}^{a}\right]-\left[\omega_{2}^{b}\right]$, which are assigned zero probability have been omitted. This leaves four histories: two possibilities at $t_{2}$ and two at $t_{3}$. To these the extended Born rule assigns probabilities:

$$
\operatorname{Pr}\left(\omega_{2}^{a}, D_{3}^{a *}\right)=|\alpha|^{2}, \quad \operatorname{Pr}\left(\omega_{2}^{a}, D_{3}^{b *}\right)=0 ; \quad \operatorname{Pr}\left(\omega_{2}^{b}, D_{3}^{b *}\right)=|\beta|^{2}, \quad \operatorname{Pr}\left(\omega_{2}^{a}, D_{3}^{a *}\right)=0,
$$

where the subscript 3 has been added to the detector states to indicate the time at which this property is or is not true. Combining (13) with (8) yields the conditional probabilities:

$$
\operatorname{Pr}\left(\omega_{2}^{a} \mid D_{3}^{a *}\right)=1, \quad \operatorname{Pr}\left(\omega_{2}^{b} \mid D_{3}^{b *}\right)=1 .
$$

Thus if at $t_{3} D^{a}$ has triggered one can be certain (conditional probability 1 ) that at $t_{2}$ the particle was earlier on the path $a$ in Fig. 1 leading to this detector, or on path $b$ if $D^{b}$ has triggered. An experimental physicist who has designed this type of equipment will find these conclusions very reasonable.

The discussion of particle properties can be extended to a still earlier time by using the family

$$
\mathcal{F}_{3}:\left[\Psi_{0}\right] \odot\left\{\left[z^{+}\right],\left[z^{-}\right]\right\} \odot I \odot\left\{\left[D^{a *}\right] \otimes\left[D^{b}\right],\left[D^{a}\right] \otimes\left[D^{b *}\right]\right\},
$$

where now the spin states that appear at $t_{1}$. Repeating the sort of analysis applied to $\mathcal{F}_{2}$ leads to the conclusion that

$$
\operatorname{Pr}\left(\left[z^{+}\right]_{1} \mid D_{3}^{a *}\right)=1, \quad \operatorname{Pr}\left(\left[z^{-}\right]_{1} \mid D_{3}^{b *}\right)=1,
$$

where the subscript 1 has been added to $\left[z^{+}\right]$and $\left[z^{-}\right]$to emphasize that these properties refer to time $t_{1}$, i.e., before the particle entered the magnetic field gradient, see Fig. 1. As the apparatus in Fig. 1 was designed to measure $S_{z},(16)$ confirms that it operates correctly.

It is important to note that within $\mathrm{CH}$ as a formulation of the fundamental principles of quantum mechanics the frameworks $\mathcal{F}_{u}, \mathcal{F}_{1}, \mathcal{F}_{2}, \mathcal{F}_{3}$ are all equally valid quantum descriptions, and there is no principle of quantum physics which demands the use of one rather than another. What distinguishes the different frameworks is their utility in describing the quantum system of interest and drawing physical conclusions about it. Thus while $\mathcal{F}_{u}$ represents perfectly good physics, it cannot represent a measurement process when 'measurement' is understood in the usual way as a process resulting in one of a collection of macroscopic outcomes. Similarly, it is only $\mathcal{F}_{2}$ and $\mathcal{F}_{3}$ that can represent a quantum measurement as that term is employed in experimental physics, where an earlier microscopic property is inferred from the later macroscopic outcome. Given the multiplicity of possible frameworks one might be concerned that different choices could give rise to contradictory results, but this cannot occur, as shown in Ch. 16 of $[4]^{2}$.

\footnotetext{
${ }^{2}$ Various claims to the contrary, [34], [35] and [23] have been responded to in [36], [37] and [38], respectively; short replies by the critics follow immediately after each response.
} 


\subsection{Quasiclassical frameworks}

The preceding discussion of the measurement process has been carried out using only pure quantum states, and the reader may wonder whether this is appropriate, especially for apparatus states. Should one not use Hilbert subspaces of higher dimension, or density operators? The analysis given here is not misleading, but it can also be extended to a more general framework in which both the initial state of the apparatus and its final pointer states have a quasiclassical description. That is to say, one in which those macroscopic quantities that one expects will be correctly described using classical mechanics are represented by appropriate Hilbert subspaces of enormous dimension, in such a way that the stochastic quantum dynamics of the associated family of histories is in turn well approximated by the deterministic dynamical laws of classical physics. While the emergence of classical mechanics from quantum mechanics in this way has not been rigorously demonstrated, there is significant work indicating how it can be done. We refer the reader to [18,39], and to Ch. 17 of [4] for a specific application to measuring processes.

In this connection it is important to note that as a statement of fundamental quantum principles, $\mathrm{CH}$ does not require the use of quasiclassical frameworks in situations where they might seem to be a natural choice, as at time $t_{3}$ in the Stern Gerlach measurement. As noted above, the framework $\mathcal{F}_{u}$ is perfectly acceptable as a quantum description, though not very useful for describing a measurement. Similarly, in other situations where quantum mechanics supplies a quasiclassical description there are always other frameworks which are very far from being quasiclassical, a fact that has sometimes been used to declare the $\mathrm{CH}$ approach unacceptable [22, 40,41]; see the discussion in Sec. 4.2 of [19].

\section{Alternative Approaches to the Second Measurement Problem}

\subsection{Standard (textbook) quantum mechanics}

Let us begin a discussion of other approaches to the second measurement problem by considering standard quantum mechanics i.e., what is found in textbooks or based thereon. In textbooks a typical discussion of measurements will contain the following elements:

M1. If a quantum system is initially in an eigenstate of a particular observable, a measurement of that observable will with certainty give the corresponding eigenvalue.

M2. If a quantum system is not initially in an eigenstate, then the measurement outcome will correspond to one of the observable's eigenvalues, with a probability given by the Born formula.

M3. In case M2 the measurement will cause the measured system to jump or collapse into an eigenstate corresponding to the measured eigenvalue.

The Hermitian operator $A$ representing a quantum observable has a spectral representation

$$
A=\sum_{j} a_{j} P_{j}
$$

where the $\left\{P_{j}\right\}$ are a PDI, and we assume each eigenvalue appears only once in the sum: $j \neq k$ implies $a_{j} \neq a_{k}$. Hence $P_{j}$ projects on the subspace spanned by all the eigenvectors with eigenvalue $a_{j}$. Thus a measurement of $A$ that yields the value $a_{j}$ is equivalent to a measurement of the corresponding PDI that reveals the property $P_{j}$.

The $\mathrm{CH}$ discussion in Sec. 4 of the situation represented in Fig. 1 is consistent with M1 and M2. (Since M3 only applies to the case of special sort of nondestructive measurement which can be thought of as combining a preparation with a measurement, see Sec. 2, it will not be discussed further here; see [20, 21] for a $\mathrm{CH}$ treatment of such measurements.) If one somehow knows that the system was initially in some eigenstate of the measured observable, then by $\mathbf{M} 1$ the measurement outcome can be used to infer this state before the measurement took place, but for a general superposition state such an inference is not possible. This is because the textbook approach corresponds to using the family $\mathcal{F}_{1}$ of Sec. 5.1 when analyzing the measurement in Fig. 1: unitary time development until the final pointer position emerges.

From the $\mathrm{CH}$ perspective the textbook discussion is not incorrect, but it is inadequate, and the inadequacy is not entirely trivial, as experimental physicists often infer something about the preceding trajectory of a particle from the path revealed by their detectors. Suppose that a particle is scattered in an S (spherically 
symmetrical) wave from a target, and is detected some distance away by a detector preceded by a collimator, a metal plate with a small hole in it, that serves to define the precise scattering angle. When a particle is detected the experimenter makes the seemingly reasonable inference that it followed a straight line from some point on the target and passed through the hole in the collimator on its way to the detector. The $\mathrm{CH}$ approach allows the use of a framework in which the particle emerges from the scattering center in a spherical wave, but also a different framework, incompatible with the first one, with histories in which the particle travels outwards from the target as a wavepacket in a definite (but not infinitely precise) direction. This second framework justifies the experimenter's intuition and resolves one of the standard paradoxes of quantum mechanics first pointed out by Einstein (see pp. 115f in [31]), the seemingly instantaneous "collapse" of a spherical wave upon detection.

Despite the fact that textbooks do not provide an adequate discussion of how to deduce prior states from measurement outcomes, students trained by this approach, with its emphasis on calculational techniques rather than genuine physical understanding, are nonetheless often able to draw reasonable conclusions when they think about what is going on in a particle experiment, such as the one described in the preceding paragraph, conclusions which can be justified by $\mathrm{CH}$ methods. Thus $\mathrm{CH}$ can be regarded as clarifying rather than being in direct conflict with much of what is found in textbooks, justifying the claim of its proponents that instead of being some completely new theory $\mathrm{CH}$ is "Copenhagen done right."

\subsection{Spontaneous localization and many worlds}

The spontaneous localization and many worlds approaches to the first measurement problem were discussed above in Sec. 4.2. Neither approach has a satisfactory answer to the the second measurement problem, for reasons which can be readily understood with reference to Fig. 1. Let us begin with spontaneous localization, which replaces the Schrödinger equation with a stochastic variant that causes the wavefunction to spontaneously collapse in the course of time. The collapse rate is chosen to be very slow so as not to be in obvious disagreement with existing experiments, but as a consequence when the particle emerges from the field gradient at time $t_{2}$ in Fig. 1 it will be in (or very close to) a coherent superposition of the position wave packets $\left|\omega_{2}^{a}\right\rangle$ and $\left|\omega_{2}^{b}\right\rangle$. Consequently, from the later result that, say, $D^{a}$ triggered, there is no way of inferring that the particle at $t_{2}$ was in the upper track in Fig. 1, much less the property $S_{z}=+1 / 2$ at $t_{1}$. Thus this formalism does not contain the mathematical machinery needed to support an inference which experimental physicists make very easily: the detector triggered because a particle was traveling towards it. The same criticism applies to the many worlds approach for which there is nothing at any time except for the unitarily developing Schrödinger wave. Obviously nothing in $\left|\Psi_{j}\right\rangle$ for $j=0,1,2$ gives any hint as to which detector will later detect the particle, and thus many worlds lacks a mathematical procedure for making the required inference. It is hard to see how "splitting universes" can resolve this problem unless the splitting occurs by the time the wave packet emerges from the magnetic field gradient. And that does not seem very satisfactory given that experiments exist for detecting the interference of matter waves spread out over small but still macroscopic distances.

\subsection{Bohmian mechanics}

The situation is different for Bohmian mechanics because in this interpretation a particle has a welldefined trajectory in real space, and using this trajectory makes it possible to infer from detection by $D^{a}$ that the particle was at time $t_{2}$ on the upper $a$ track in Fig. 1. However, there is another situation in which Bohmian mechanics gives an answer that does not agree with physical intuition. It is discussed by Bell, see Ch. 14 of [42], using a gedanken experiment inspired by Wheeler [43], a variant of the well-known double slit situation shown here schematically in Fig. 2. The Schrödinger wave for a single particle arrives from the left as a plane wave, and the part of the wave that passes through the $A B$ slit system is refracted by passive elements so that the resulting beams are bent and cross each other before traveling on to the two detectors $C^{a}$ and $C^{b}$. The $X$ in the figure labels the position in the vacuum where the beams cross; there is nothing at this point for the particle to interact with. Unlike the wave, the Bohmian particle has a precise position at every time and follows a continuous trajectory in space; which trajectory it follows depends on its initial position, which is random. Some trajectories pass through the upper and some through the lower slit. Assume the particle is eventually detected by detector $C^{a}$. Did it earlier pass through the upper slit $A$ or the lower slit $B$ ? When asked this question a physicist is likely to reply $B$, an answer which Bell dismisses as resulting from a "naive classical picture." The answer given by Bohmian mechanics is $A$, 
because the quantum wave function is symmetric under a reflection that interchanges the two slits, and this means the Bohmian particle trajectory can never cross the symmetry plane midway between the slits, from top to bottom or vice versa. Indeed, an analysis of Bohmian particle trajectories shows that those that come through slit $A$ and descend downwards towards $X$ eventually "bounce" back upwards towards $C^{a}$, while those coming through $B$ bounce downwards and eventually reach $C^{b}$.

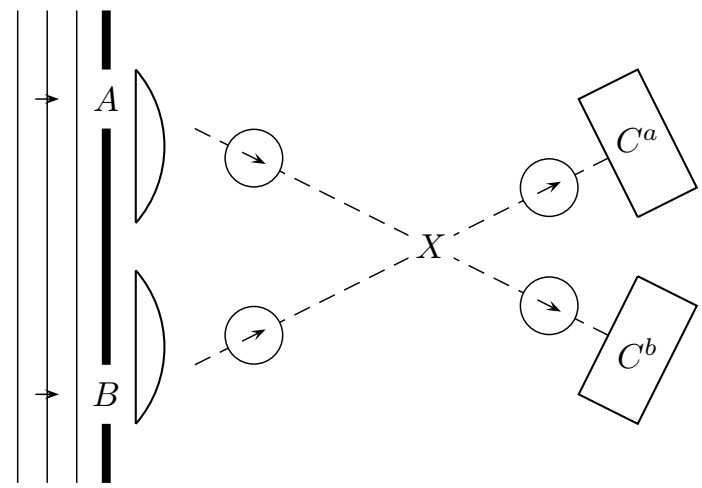

Figure 2: Double slit interference with bent beams that cross at $X$ and are later detected by $C^{a}$ and $C^{b}$. The circles represent wave packets before and after they cross at $X$.

However, the $\mathrm{CH}$ analysis [44] of this situation, which is fully quantum mechanical, when addressing the question of which slit a particle detected by $C^{a}$ had earlier passed through gives the answer $B$, contrary to Bohmian mechanics, but in agreement with naive classical thinking. The particle passes through $X$ without a bounce. In addition, a numerical study by Englert et al. [45] showed that if a certain type of nondestructive detector $N$ (not shown in Fig. 2 ) that is triggered by a particle passing through it is placed on the lower path between $B$ and $X$, it registers the particle's passage in coincidence with $C^{a}$ _either both are triggered, or neither. However, when this situation is analyzed using Bohmian mechanics, it is sometimes the case that the Bohmian particle follows the upper path on its way to $C^{a}$, and nonetheless the detector $N$ is triggered. From this Englert et al. concluded that the Bohmian particle trajectory is "surrealistic", without physical significance. That Bohmian trajectories can have the strange behavior pointed out in [45] was later confirmed through careful numerical calculations by Dewdney et al. [46] who, unlike the authors of [45], were in favor of, or at least not critics of, Bohmian mechanics. The supporters of Bohmian mechanics have defended their claim that the bouncing particle trajectory is physically plausible by, among other things, asserting that standard quantum mechanics cannot say anything about the position of a particle prior to its measurement, or is at best ambiguous [47,48]. But the $\mathrm{CH}$ analysis in [44], which extends standard quantum mechanics in an unambiguous way that resolves the second measurement problem, indicates that $N$ will be triggered if and only if the particle passes through it, a result which most physicists would consider reasonable. For further details the reader is referred to [44] and the references given there. The conclusion is that the answer Bohmian mechanics gives to the second measurement problem is not always credible.

\section{The Criticisms of Okon and Sudarsky}

Now that the application of the $\mathrm{CH}$ approach to a particular measurement situation has been discussed in some detail and compared with certain other quantum interpretations, including spontaneous localization and Bohmian mechanics, both of which Okon and Sudarsky consider superior to CH, we are in a position to address the criticisms found in [1]. Mainly these concern how $\mathrm{CH}$ approaches the first measurement problem, with some additional remarks about how it resolves the second problem.

\subsection{First measurement problem}

The principal criticism in [1] of the $\mathrm{CH}$ approach to the first measurement problem will be evident from the following quotations. (Here an ' $a$ ' and ' $b$ ' following a page number indicate the first and second column, respectively.) 
Q1. We find [the CH treatment of measurements] unsatisfactory because it relies, often implicitly, on elements external to those provided by the formalism. In particular, we note that, in order for the formalism to be informative when dealing with measurement scenarios, one needs to assume that the appropriate choice of framework is such that apparatuses are always in states of well defined pointer positions after measurements. The problem is that there is nothing in the formalism to justify this assumption. (Abstract)

Q2. [A]mong all the possible frameworks, only one is suitable to describe what in fact we perceive or experience...[T]he main problem is that $\mathrm{CH}$ is incapable of recognizing in advance, and without bringing in elements that rely on our intuition and experience, which is going to be the framework that does the job. We conclude, then, that $\mathrm{CH}$ does not really constitute a satisfactory solution to the measurement problem. (Sec. 3, p. 9b)

Q3. [T] he fact that a given measuring apparatus actually measures some property is something that cannot be deduced from the $\mathrm{CH}$ formalism but that must be discovered by experience. That is, if one is given a new measurement equipment, described entirely in quantum terms (via a Hamiltonian, an initial state, etc.), $\mathrm{CH}$ is unable to answer, unlike, say [spontaneous localization] or Bohmian mechanics, which are going to be its possible final states when we use it in the laboratory. Therefore, in a given situation, described by providing the complete physical set-up in terms of the initial state of the closed system and the Hamiltonian, $\mathrm{CH}$ is incapable of predicting which framework one must choose. (Sec. 3, p. 10b)

Q4. [T] he truly problematic point... is that the $\mathrm{CH}$ formalism is incapable of picking out the right framework. That is, it does not offer any clear characterization regarding the exact relation between the experimental set-up and the framework one needs to use in order to get reliable predictions out of the theory. (Sec. 3, p. 11a)

In response to the assertion in $\mathbf{Q 1}$ that $\mathrm{CH}$ employs elements external to the formalism when discussing measurements, we note that this is quite proper insofar as $\mathrm{CH}$ is a statement of the fundamental principles of quantum mechanics. Indeed, most physicists working in quantum foundations do not think that 'measurement' should be included among the fundamental principles, and surely one of the main motivations behind the spontaneous localization, Bohmian mechanics, and many worlds approaches is to find principles that do not refer to measurements. To use an analogy, one can employ the fundamental principles of optics, such as refraction and reflection, to describe the operation of a telescope, but it would be extremely odd to find 'telescope' playing an essential role among the principles of optics, which apply equally to microscopes, eyeglasses, the human eye, and so on. Thus in the case of quantum measurements one would anticipate that any plausible fundamental theory of quantum mechanics would contain no reference to them, and therefore additional concepts must be employed in order to to describe them. The proper question is not whether elements external to the formalism are used, but instead whether these "extras" are needed because one is discussing a measurement and not some other physical process.

To be specific, the complaint in Q1 is that $\mathrm{CH}$ must choose a framework in which the apparatus has suitable outcome states, pointer positions, at the end of the measurement. That is correct: in the $\mathrm{CH}$ approach all four families of histories introduced in Sec. 5.1 represent valid quantum descriptions of the situation shown in Fig. 1, but only the last three include the pointer positions, and hence constitute possible descriptions of a measurement understood as a physical process in which prior microscopic properties are later revealed or correlated with macroscopic outcomes. A framework that makes no reference to the microscopic properties or to the macroscopic outcomes cannot describe a 'measurement' as that term is understood in experimental quantum physics, any more than a discussion omitting all reference to its mirror and the image it produces could be said to describe a reflecting telescope. Since macroscopic properties are described in the $\mathrm{CH}$ approach using a quasiclassical framework, Sec. 5.2, and the superposition state $\left|\Psi_{3}\right\rangle$ does not belong to such a framework, it cannot possibly be used when describing a measurement, a point nowhere better stated than in Bell [28]. In thus excluding $\mathcal{F}_{u}$ from the collection of frameworks which might describe a measurement, $\mathrm{CH}$ is employing the single framework rule, which is very much part of its formalism, along with the notion of a quasiclassical framework which, although not a fundamental quantum principle, is what it uses to describe the macroscopic world. To be sure, this is very different from the paths followed by spontaneous localization (modified Schrödinger dynamics) and Bohmian mechanics (use of particle positions and dynamics in addition to the Hilbert space) in their approach to the first measurement problem. But 
that it is different does not mean it is wrong or inadequate or inferior or incomplete, as Q3 would seem to imply.

Turning to Q2, the topic of what human beings can perceive and experience is a fairly subtle one, and from the quantum perspective not free from controversy. However, if one is willing to allow that those perceptions and experiences most relevant to the present discussion can be described using a quasiclassical framework, then the response can be similar to that given above for Q1: only quasiclassical properties and processes will be of use in a quantum description of something people can perceive, and this fact can again be used to rule out $\mathcal{F}_{u}$, with its far-from-quasiclassical $\left|\Psi_{3}\right\rangle$, as appropriate for describing a measurement whose outcome can be seen and discussed by scientists.

What seems really at stake in Q3 is the fact that spontaneous localization and Bohmian mechanics, both of which are stochastic, employ sample spaces which are fixed in advance by the postulates of the theory, whereas $\mathrm{CH}$ does not specify the sample space in advance: each of the frameworks introduced in Sec. 5.1 is a separate sample space, and they are mutually incompatible so they cannot be combined. This structure is certainly distinct from anything one finds in classical physics, and the final statement in Q3, that the $\mathrm{CH}$ approach is "incapable of predicting which framework one must choose" is correct. The choice of a framework is one made by the physicist constructing a quantum description, and there is no constraint among the principles of $\mathrm{CH}$ that prescribes which one must be used. However, the consistent historian will add that if the "given measuring apparatus actually measures some property," that fact alone is enough to constrain the choice of an appropriate framework to one such as $\mathcal{F}_{2}$ or $\mathcal{F}_{3}$ among those discussed in Sec. 5.1, contrary to the claim in the final sentence in Q3. And it is this kind of "utilitarian" consideration that allows $\mathrm{CH}$ to solve the second measurement problem, one which, see Sec. 6, standard quantum mechanics, spontaneous localization, Bohmian mechanics, and many worlds are unable to address.

As for Q4 the discussion given in Secs. 4 and 5, together with the comments above about Q1, Q2, and Q3, should constitute a sufficient answer to the second sentence, and need not be repeated. But the first sentence, the assertion that "the $\mathrm{CH}$ formalism is incapable of picking out the right framework" deserves additional comment. The notion that there must be a single "right" framework comes from the classical world, from classical physics, where it is assumed, often implicitly, that at any given time there is a single true state of the world. In Sec. 27.3 of [4] this notion is given a technical name: unicity. From a physicist's perspective unicity is exemplified by the fact that in a classical phase space a single point represents the exact and true state of the system at a particular time; all properties of the system represented by sets of points that include this point are true, and those that do not include this point are false. By contrast, in a Hilbert space there is no single subspace with a comparable property. Hence if a Hilbert space, rather than a classical phase space or a classical collection of hidden variables, provides the correct mathematical description of physical reality, one might expect the principle of unicity to fail, and thus in an absolute sense there is no "right framework."

\subsection{Second measurement problem}

The criticism in [1] of the $\mathrm{CH}$ approach to the second measurement problem is relatively brief. After citing a particular example from [4] the authors comment that:

Q5. It seems... that one can say that some particle had a definite property before a measurement took place only if one chooses a specific framework where this is so. But, if according to $\mathrm{CH}$, all frameworks are equally valid, it is not clear why the description according to this one framework should be taken that seriously. That is, the problem is solved in only one of an infinite number of possible frameworks.

Following this is a footnote in which they claim that d'Espagnat [49] in 1987 provided a stronger argument against the possibility of $\mathrm{CH}$ solving the second measurement problem.

Let us begin with Q5. It is indeed the case that one can only discuss the property a particle had before the measurement by choosing a specific framework that includes the property in question at the appropriate time. Thus it is necessary to use $\mathcal{F}_{3}$ in Sec. 5.1, or some other framework which includes the same possibilities at $t_{1}$, in order to discuss whether the measurement outcome tells one the value of $S_{z}$ before the measurement took place. This should not be surprising; in the $\mathrm{CH}$ approach quantum properties can only be discussed when they appear in a suitable framework, one that contains the appropriate projectors as part of the event algebra. We have already discussed this in some detail with reference to measurement outcomes in Sec. 7.1, and exactly the same principle applies to measured properties. The assertion in Q5 that because there are alternative frameworks the result obtained using the $S_{z}$ framework "should not be taken seriously" seems 
extremely odd. Is a news account stating that it was snowing on a particular day in Pittsburgh rendered invalid by an alternative news account which describes actions by the city council on the same day, but makes no mention of snow? Were it the case that different quantum frameworks gave contradictory results, this would indeed be a cause for concern. But they do not; the consistency of CH inferences is guaranteed by the considerations given in Ch. 16 of [4]. Thus the existence of other frameworks is entirely beside the point, as it no way invalidates the description obtained using a particular one. There are also an infinite number of possible frameworks for discussing the situation at time $t_{3}$ in Fig. 1, but most of them do not allow any discussion of the measurement outcome as having definite results, without which there is no reason to call it a measurement. Similarly, choosing a framework in which the quantum properties the measurement was designed to measure cannot even be discussed disqualifies using the term "measurement" as that is normally employed in experimental physics, and as it ought to be employed in discussions of quantum foundations.

The 1987 paper by d'Espagnat [49] contains two arguments which might be considered as counting against the $\mathrm{CH}$ solution of the second measurement problem. It focuses on the property of a spin-half particle at a time $t_{1}$ between when it is prepared at time $t_{0}$ and when it is measured at time $t_{2}$. The first argument requires combining two incompatible frameworks in a manner forbidden by the single framework rule, which had not been as clearly formulated in 1987 as it was later. For an extended response to this and similar criticisms by d'Espagnat see Sec. V B of [50]. The second argument involves what would happen if a different measurement were carried out at time $t_{2}$, and can be seen as a version of Wheeler's "delayed choice" experiment [43] involving counterfactual considerations: something was measured but something else could have been measured. Counterfactuals and delayed choice experiments are discussed in considerable detail in Chs. 19 and 20 of [4], where it is shown that the $\mathrm{CH}$ treatment is both consistent and coherent. For a more recent discussion involving counterfactuals see the exchange between Stapp and the author $[51,52]$.

\section{Conclusion}

With reference to a particular Stern Gerlach measurement situation introduced in Sec. 3 we have shown in Secs. 4 and 5 how the consistent histories $(\mathrm{CH})$ approach resolves both parts of the quantum measurement problem as defined in Sec. 2, allowing a measurement, a macroscopic output revealing a prior microscopic quantum property, to be discussed from beginning to end in fully quantum mechanical terms. This requires the introduction of appropriate frameworks or quantum sample spaces, and the use of the extended Born rule to assign probabilities with the help of (unmodified) Schrödinger dynamics. The main innovation of the $\mathrm{CH}$ approach relative to standard (textbook) quantum mechanics is the use of a fully consistent system of probabilities at both the microscopic and macroscopic levels. This allows a quantum system to be described using a variety of different frameworks, but then insists (the single framework rule) that incompatible frameworks cannot be combined.

Four of a large number of possible frameworks for the measurement situation shown in Fig. 1 and described in Sec. 3 are discussed in Sec. 5.1, and they illustrate the fact that only certain frameworks are suitable for discussing this measurement as a measurement, using that term in the way it is employed by physicists who conduct actual experiments. In particular, an acceptable framework should include both microscopic properties before the measurement takes place and macroscopic outcomes at its conclusion. It is much to be regretted that the longstanding confusion surrounding the measurement problem in quantum foundations has led to a focus on the problem of understanding measurement outcomes in quasiclassical terms (getting rid of Schrödinger's cat), the first measurement problem in the terminology used in this paper, to the neglect of addressing the equally important question of how this outcome is related to the microscopic property that was (supposedly) measured, in our terminology the second measurement problem. Both problems must be resolved if one wants to claim that experimental results of measurements confirm the correctness of quantum theory. At present $\mathrm{CH}$ appears to be the only quantum interpretation that resolves the second problem.

The principal criticisms of Okon and Sudarsky [1], see Sec. 7.1, when applied to the example in Sec. 3 as analyzed from the $\mathrm{CH}$ perspective in Secs. 4 and 5 , has to do with choosing one of the frameworks $\mathcal{F}_{1}, \mathcal{F}_{2}$, or $\mathcal{F}_{3}$ in Sec. 5.1, rather than $\mathcal{F}_{u}$, in order to describe the measurement outcome. These authors consider the CH approach, which rejects a family of histories containing $\left|\Psi_{3}\right\rangle$ at time $t_{3}$ because it cannot represent a quantum property corresponding to a macroscopic measurement outcome, as inadequate, or at least inferior to the spontaneous localization and Bohmian mechanics procedures for resolving the first measurement problem. Our response, given in detail in Sec. 7.1, is that the CH approach employs its own principles correctly, and does not go outside of its formalism except to include features specific to a measurement as that term 
is understood by physicists, and hence necessary for this particular application of these principles. As a fundamental theory of quantum mechanics $\mathrm{CH}$ makes no reference to measurements, which is one reason it can be considered an advance over standard (textbook) quantum theory.

These authors also criticize the CH solution to the second measurement problem, Sec. 7.2, again on the grounds that there is no way within the formalism of identifying the correct framework. Our response, as before, is that if a measurement is supposed to have measured some microscopic quantum property, the quantum description of the measurement process must necessarily include that property at a time before the measurement took place, and this suffices for identifying an appropriate framework. As noted in Sec. 6.2, it seems clear that neither the spontaneous localization nor the many worlds interpretations possess the means to resolve the second measurement problem, whereas Bohmian mechanics does not always give the right answer, i.e., a solution that working physicists would consider plausible. In addition, comments on some out-of-date criticisms of CH by d'Espagnat have been placed at the end of Sec. 7.2.

Although there is no discussion of it in [1], it seems likely that fundamental to the Okon and Sudarsky criticism is their adherence to the concept of unicity, the idea that at any single time the world is described by precisely one mechanical state. As noted in Sec. 7.2 , this idea fits very well with classical mechanics, where a single point in its phase space provides a complete characterization of a mechanical system, but is incompatible with the structure of Hilbert space. The fact that from the quantum perspective a single quasiclassical framework suffices for all of classical physics provides a plausible explanation of why the concept of unicity is so deeply embedded in human thinking, and thus why it is so difficult to accept the notion that at the quantum level there are multiple incompatible frameworks and unicity no longer holds. If the $\mathrm{CH}$ approach is correct then, as noted in Sec. 1, unicity in the modern context is somewhat analogous to the notion of an immobile earth, which had to be discarded with the rise of modern cosmology, and thus abandoning unicity is necessary in order to reach a proper understanding of the quantum world. Unicity is also maintained in the spontaneous localization, Bohmian mechanics, and many worlds interpretations, and it is what separates them from $\mathrm{CH}$ at the most fundamental level.

\section{Acknowledgments}

I thank F. Laloë and P. Pearle for helpful correspondence. The research described here received support from the National Science Foundation through Grant 1068331.

\section{References}

[1] Elias Okon and Daniel Sudarsky. Measurements according to consistent histories. Stud. Hist. Phil. Mod. Phys., 48:7-12, 2014. arXiv:1309.0792.

[2] Robert B. Griffiths. Quantum locality. Found. Phys., 41:705-733, 2011. arXiv:0908.2914.

[3] Robert B. Griffiths. Hilbert space quantum mechanics is noncontextual. Stud. Hist. Phil. Mod. Phys., 44:174-181, 2013. arXiv:1201.1510.

[4] Robert B. Griffiths. Consistent Quantum Theory. Cambridge University Press, Cambridge, U.K., 2002. http://quantum.phys.cmu.edu/CQT/.

[5] G. C. Ghirardi, A. Rimini, and T. Weber. A model for a unified quantum description of macroscopic and microscopic systems. In L. Accardi and W. von Waldenfeis, editors, Quantum Probability and Applications II, Lecture Notes in Mathematics, pages 223-232. Springer-Verlag, Berlin, 1985.

[6] G. C. Ghirardi, A. Rimini, and T. Weber. Unified dynamics for microscopic and macroscopic systems. Phys. Rev. D, 34:470-491, 1986.

[7] Philip Pearle. Combining stochastic dynamical state-vector reduction with spontaneous localization. Phys. Rev. A, 39:2277-2289, 1989.

[8] Roman Frigg. GRW theory (Ghirardi, Rimini, Weber model of quantum mechanics). In Daniel Greenberger, Klaus Hentschel, and Friedel Weinert, editors, Compendium of Quantum Physics, pages 266-270. Springer-Verlag, Berlin, 2009. 
[9] GianCarlo Ghirardi. Collapse theories. Stanford Encyclopedia of Philosophy, 2011. http://plato.stanford.edu/entries/qm-collapse/.

[10] L. de Broglie. La mécanique ondulatoire et la structure atomique de la matière et du rayonnement. $J$. Physique Radium, 8:225-241, 1927.

[11] David Bohm. A suggested interpretation of the quantum theory in terms of "hidden" variables. I. Phys. Rev., 85:166-179, 1952.

[12] Peter R. Holland. The Quantum Theory of Motion. Cambridge University Press, Cambridge, 1993.

[13] Sheldon Goldstein. Bohmian mechanics. Stanford Encyclopedia of Philosophy, 2012. http://plato.stanford.edu/entries/qm-bohm/.

[14] Hugh Everett III. "Relative state" formulation of quantum mechanics. Rev. Mod. Phys., 29:454-462, 1957.

[15] Bryce S. DeWitt and Neill Graham, editors. The Many-Worlds Interpretation of Quantum Mechanics. Princeton University Press, Princeton, N.J., 1973.

[16] Simon Saunders, Jonathan Barrett, Adrian Kent, and David Wallace, editors. Many Worlds? Everett, Quantum Theory, 8 Reality. Oxford University Press, Oxford, 2010.

[17] Robert B. Griffiths. Consistent Histories. In Daniel Greenberger, Klaus Hentschel, and Friedel Weinert, editors, Compendium of Quantum Physics, pages 117-122. Springer-Verlag, Berlin, 2009.

[18] James B. Hartle. The quasiclassical realms of this quantum universe. Found. Phys., 41, 982-1006, 2011.

[19] Robert B. Griffiths. A consistent quantum ontology. Stud. Hist. Phil. Mod. Phys., 44:93-114, 2013. arXiv:1105.3932.

[20] Robert B. Griffiths. The New Quantum Logic. Found. Phys., 44:610-640, 2014. arXiv:1311.2619 v2.

[21] Robert B. Griffiths. The Consistent Histories Approach to Quantum Mechanics. Stanford Encyclopedia of Philosophy, 2014. http://plato.stanford.edu/entries/qm-consistent-histories/.

[22] Adrian Kent. Quantum histories. Physica Scripta, T76:78-84, 1998.

[23] Angelo Bassi and GianCarlo Ghirardi. Decoherent histories and realism. J. Stat. Phys., 98:457-494, 2000 .

[24] Philip Pearle. Completely quantized collapse and consequences. Phys. Rev. A, 72:022112, 2005.

[25] N.David Mermin. Annotated interview with a QBist in the making. arXiv:1301.6551, 2013.

[26] Otto Stern. Ein Weg zur experimentellen Prüfung der Richtungsquantelung im Magnetfeld. Z. Phys., 7:249-253, 1921.

[27] Walther Gerlach and Otto Stern. Der experimentelle Nachweis der Richtungsquantelung im Magnetfeld. Z. Phys., 9:349-352, 1922.

[28] J. S. Bell. Against measurement. In Arthur I. Miller, editor, Sixty-Two Years of Uncertainty, pages 17-31. Plenum Press, New York, 1990.

[29] Stephen L. Adler. Why decoherence has not solved the measurement problem: a response to P. W. Anderson. Stud. Hist. Phil. Mod. Phys., 34:135-142, 2003.

[30] G. Birkhoff and J. von Neumann. The logic of quantum mechanics. Ann. Math., 37:823-843, 1936.

[31] Max Jammer. The Philosophy of Quantum Mechanics. Wiley, New York, 1974.

[32] Max Born. Zur Quantenmechanik der Stoßvorgänge. Z. Phys., 37:863-867, 1926.

[33] Max Born. Quantenmechanik der Stoßvorgänge. Z. Phys., 38:803-827, 1926. 
[34] Adrian Kent. Consistent sets yield contrary inferences in quantum theory. Phys. Rev. Lett., 78:28742877, 1997.

[35] Angelo Bassi and GianCarlo Ghirardi. Can the decoherent histories description of reality be considered satisfactory? Phys. Lett. A, 257:247-263, 1999.

[36] Robert B. Griffiths and James B. Hartle. Comment on "Consistent sets yield contrary inferences in quantum theory". Phys. Rev. Lett., 81:1981, 1998.

[37] Robert B. Griffiths. Consistent histories, quantum truth functionals, and hidden variables. Phys. Lett. $A, 265: 12-19,2000$.

[38] Robert B. Griffiths. Consistent quantum realism: A reply to Bassi and Ghirardi. J. Stat. Phys., 99:1409-1425, 2000.

[39] Murray Gell-Mann and James B. Hartle. Classical equations for quantum systems. Phys. Rev. D, 47:3345-3382, 1993.

[40] Fay Dowker and Adrian Kent. On the consistent histories approach to quantum mechanics. J. Stat. Phys., 82:1575-1646, 1996.

[41] Adrian Kent. Quasiclassical dynamics in a closed quantum system. Phys. Rev. A, 54:4670-4675, 1996. arXiv:gr-qc/9512023v3.

[42] J. S. Bell. Speakable and Unspeakable in Quantum Mechanics. Cambridge University Press, Cambridge, 1987.

[43] John Archibald Wheeler. The "Past" and the "Delayed-Choice" Double-Slit Experiment. In A. R. Marlow, editor, Mathematical Foundations of Quantum Theory, pages 9-48. Academic Press, New York, 1978.

[44] Robert B. Griffiths. Bohmian mechanics and consistent histories. Phys. Lett. A, 261:227-234, 1999. quant-ph/9902059.

[45] Berthold-Georg Englert, Marlan O. Scully, Georg Süssmann, and Herbert Walther. Surrealistic Bohm trajectories. Z. Naturforsch., 47a:1175-1186, 1992.

[46] C. Dewdney, L. Hardy, and E. J. Squires. How late measurements of quantum trajectories can fool a detector. Phys. Lett. A, 184:6-11, 1993.

[47] Detlef Dürr, Walter Fusseder, Sheldon Goldstein, and Nino Zanghí. Comment on "surrealistic Bohm trajectories". Z. Naturforsch., 48a:1261-1262, 1993.

[48] Peter Holland. The roads not taken: empty waves, wavefunction collapse and protective measurement in quantum theory. In Shan Gao, editor, Protective Measurement and Quantum Reality. Cambridge University Press, 2015. arXiv:1409.5817.

[49] Bernard d'Espagnat. Consistent histories and the measurement problem. Phys. Lett. A, 124:204-206, 1987.

[50] Robert B. Griffiths. Choice of consistent family, and quantum incompatibility. Phys. Rev. A, 57:16041618, 1998. quant-ph/9708028.

[51] Henry P. Stapp. Quantum locality? Found. Phys., 42:647-655, 2012. arXiv:1111.5364.

[52] Robert B. Griffiths. Quantum counterfactuals and locality. Found. Phys., 42:674-684, 2012. arXiv:1201.0255. 